Bryn Mawr College

Scholarship, Research, and Creative Work at Bryn Mawr

College

Physics Faculty Research and Scholarship

Physics

1977

\title{
Nuclear spin relaxation and centrifugal distortion effects in dilute silane gas
}

Peter A. Beckmann

Bryn Mawr College, pbeckman@brynmawr.edu

E. E. Burnell

Let us know how access to this document benefits you.

Follow this and additional works at: https://repository.brynmawr.edu/physics_pubs

Part of the Physics Commons

\section{Custom Citation}

Beckmann, Peter A. and E. E. Burnell. 1977. "Nuclear spin relaxation and centrifugal distortion effects in dilute silane gas." Canadian Journal of Physics 55.15: 1354-1355.

This paper is posted at Scholarship, Research, and Creative Work at Bryn Mawr College. https://repository.brynmawr.edu/physics_pubs/119

For more information, please contact repository@brynmawr.edu. 
Nuclear spin relaxation and centrifugal distortion effects in dilute silane gas

Peter Beckmann and E Elliott Burnell

Canadian Journal of Physics, 1976, 54.22: 2209-2212. https://doi.org/10.1139/p76-264

Abstract: We have measured the proton ioiigitudinal spin relaxation rate in dilute gaseous silane $\left(\mathrm{SiH}_{4}\right.$ between $10^{-2}$ and I amagats and are able to observe the influence of rotational $Q$ branch centrifugal distortion transitions.

In order to interpret nuclear spin relaxation measurements in dilute methane $\left(\mathrm{CH}_{4}\right)$ gas, the effects of $Q$ branch rotational centrifugal distortion transitions had to be taken into account. This was first done phenomenologically (Beckmann et al. (1972), henceforth referred to as BBB) and, subsequently, in terms of a detailed relaxation theory in which the rotational structure of the methane molecule was explicitly taken into account (Beckmann et al. (1976), henceforth referred to as BBO). In this paper we wish to report measurements of the longitudinal relaxation rate $\mathrm{T}_{1}{ }^{-1}$ as a function of density in dilute silane $(\mathrm{SiH} 4)$ gas at room temperature. The results are shown in Fig. 1 and the reader is referred to $\mathrm{BBB}$ and $\mathrm{BBO}$ for a discussion of the nuclear magnetic resonance background, the details of the experimental apparatus and technique, and the role of centrifugal distortion. The major drawback to the measurements in Fig. 1 is that we have not gone to sufficiently low density to properly characterize the $\mathrm{T}_{1}{ }^{-1}$ maximum. Since these measurements were performed, however, the rotational structure of the silane molecule has been investigated in detail by Ozier et al (1976), and we feel it important to note that this structure manifests itself in the relaxation experiments.

The expression for $\mathrm{T}_{1}{ }^{-1}$ which incorporates the effects of centrifugal distortion in a phenomenological way is given by $\mathrm{BBB}$ (see $\mathrm{BBO}$ also),

$$
\text { [1] } \begin{aligned}
T_{\mathrm{t}}^{-1}=\frac{2 \pi^{2} k T}{B_{0} h c}\left\{C_{\mathrm{a}}{ }^{2} \frac{2 \tau}{1+\omega_{0}{ }^{2} \tau^{2}}\right. \\
\left.+b C_{\mathrm{d}}{ }^{2} \frac{2 \tau}{1+\Omega^{2} \tau^{2}}\right\}
\end{aligned}
$$

where $B_{0}$ is the rotation constant (Ozier et al. 1976) and $C_{\mathrm{a}}$ and $C_{\mathrm{b}}$ are the scalar and tensor spin rotation coupling constants (Ozier et al. 1968). The nuclear Larmor frequency is $\omega_{0} / 2 \pi=30$ $\mathrm{MHz}$ and $\Omega / 2 \pi$ is an 'average' centrifugal distortion frequency (BBB). The correlation time $\tau$ for molecular reorientation is related to the density $\rho$ by $\tau^{-1}=\langle\sigma \nu\rangle \rho(\mathrm{BBO})$. The parameter $b$ can be taken to be $4 / 45$ (BBO).

At the density where $\tau^{-1}=\tau_{\mathrm{MAX}}{ }^{-1}=\omega_{0}$, the second term in [1] can be neglected if $\omega_{0} \ll \Omega$. Under these conditions, $\left(\mathrm{T}_{1}^{-1}\right)_{\mathrm{MAX}}$ can be calculated with no adjustable parameters since $B_{0}$ and $C_{a}$ are well known. This value is $\left(\mathrm{T}_{1}^{-1}\right)_{\mathrm{MAX}}=115 \mathrm{~s}^{-1}$ and agrees very well with the experimental value in the vicinity of 0.02 amagats. At high densities where $\omega_{0}^{2} \tau^{2}$ and $\Omega^{2} \tau^{2} \ll 1$, $\left(\mathrm{T}_{1} \tau\right)^{-1}$ is constant (i.e., $\mathrm{T}_{1}^{-1} \rho$ is constant) and the value of $\langle\sigma \mathrm{v}\rangle=1.1 \times 10^{10}$ (amagats $\left.\mathrm{s}\right)^{-1}$ is obtained from the measurements using the known values of $B_{0}, C_{\mathrm{a}}$, and $C_{\mathrm{d}}$. In turn, the procedure for determining an effective cross section for molecular reorientation can be found in $\mathrm{BBO}$. The contribution to the $C_{\mathrm{a}}$ term is, then, completely specified and is shown in curve $\{\mathrm{a}$ ) 
in Fig. 1. The only remaining parameter is $\Omega$ and $\Omega / 2 \pi=200 \mathrm{MHz}$ was used to generate curve (b) in Fig. 1. Curve (c) is (a) plus (b). The fact that $C_{\mathrm{d}}$ is only known to $\pm 40 \%$ (Ozier et al. 1968) coupled with the incompleteness, of the present results means that the values $\langle\sigma v\rangle$ and $\Omega$ are only known to about $\pm 25 \%$. It is interesting to compare the value obtained for $\Omega$. with that used to fit the methane experiments $(150<\Omega / 2 \pi<240 \mathrm{MHz})$ (BBB). The relaxation experiments are clearly not sensitive enough to determine if $\Omega$ is different for the two gases. The important feature, and indeed, the purpose of this paper, is to show that $\Omega \gg \omega_{0}$ : i.e., the centrifugal distortion transitions must be taken into account in interpreting the nuclear spin relaxation experiments. The phenomenological treatment of the problem given here is the same as that which has been used in methane (BBB); the detailed theoretical treatment of centrifugal distortion effects in methane (BBO) could now be applied to silane since the rotational structure is now known (Ozier et al. 1976). We note that at lower temperatures, the concept of an average distortion frequency becomes meaningless since individual transitions in low-lying rotational $J$ states become important (BBO). The same is true when the nuclear Larmor frequency is coincident with a distortion frequency (BBO). In fact, it may be that the proposals for doing 'relaxation rate spectroscopy' in methane (BBB, $\mathrm{BBO}$ ) might be better suited to silane since there are several important distortion transitions in the tens of megahertz region (Ozier et at. 1976). We note that to measure $T_{1}$ at densities lower than those reported here is difficult, but well within present technology (BBO).

Acknowledgments: We thank Myer Bloom for many fruitful discussions. This work was supported by the National Research Council of Canada.

BECKMANN, P. A., BLOOM, M., and BURNELL, E. E. 1972. Can. J.Phys.50, 251. BECKMANN.P. A., BLOOM, M., and OZIER, I. 1976. Can. J. Phys.54, 1712.

OZIER, I., CRAPO, L. M., and LEE, S. S. 1968. Phys. Rev. 172,63.

OZIER, I., LEES, R. M., and GERRY, M. C. L. 1976. Can. J, Phys.54, 1094. 
Figures:

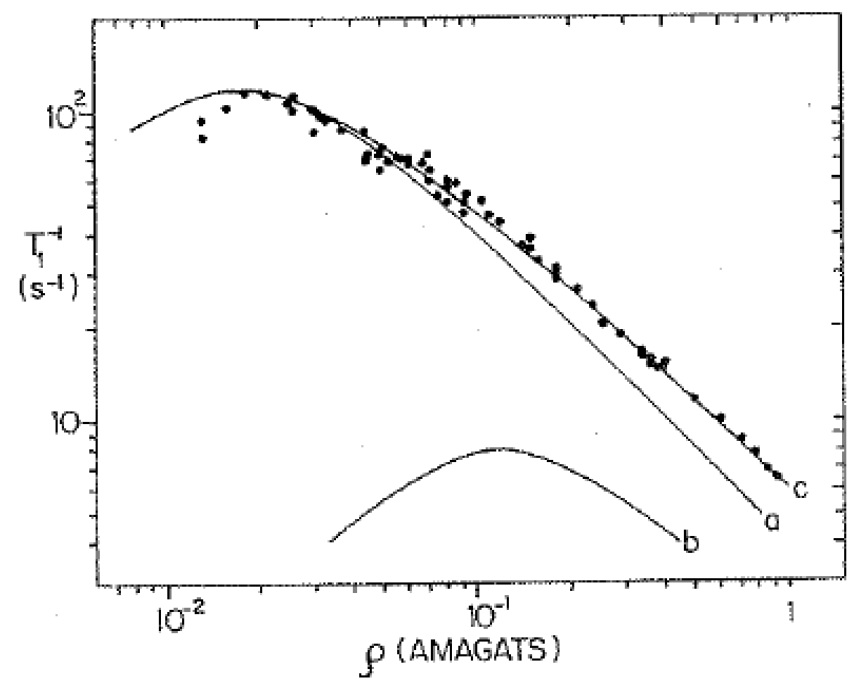

Fig. 1. The proton longitudinal relaxation rate $T_{1}^{-1}$ in gaseous $\mathrm{SiH}_{4}$ at room temperature as a function of density. Curves $(a)$ and $(b)$ represent the $C_{\mathrm{a}}$ and $C_{\mathrm{d}}$ terrns in [1] and $(c)$ is their sum. The parameters are given and discussed in the text. 